\title{
Exercise priming with transcranial direct current stimulation: a study protocol for a randomized, parallel-design, sham- controlled trial in mild cognitive impairment and Alzheimer's disease
}

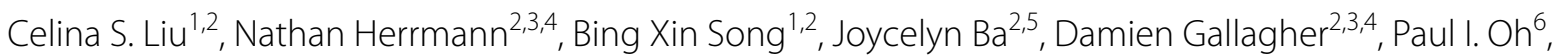
Susan Marzolini', Tarek K. Rajjij 3,7, Jocelyn Charles ${ }^{9}$, Purti Papneja9 ${ }^{9}$, Mark J. Rapoport ${ }^{2,3,4}$, Ana C. Andreazza ${ }^{1}$, Danielle Vieira ${ }^{2}$, Alex Kiss ${ }^{10}$ and Krista L. Lanctôt ${ }^{1,2,3,4,6^{*}}$

\begin{abstract}
Background: Transcranial direct current stimulation (tDCS) is a non-invasive type of brain stimulation that uses electrical currents to modulate neuronal activity. A small number of studies have investigated the effects of tDCS on cognition in patients with Mild Cognitive Impairment (MCI) and Alzheimer's disease (AD), and have demonstrated variable effects. Emerging evidence suggests that tDCS is most effective when applied to active brain circuits. Aerobic exercise is known to increase cortical excitability and improve brain network connectivity. Exercise may therefore be an effective, yet previously unexplored primer for tDCS to improve cognition in $\mathrm{MCl}$ and mild AD.

Methods: Participants with $\mathrm{MCl}$ or $\mathrm{AD}$ will be randomized to receive 10 sessions over 2 weeks of either exercise primed tDCS, exercise primed sham tDCS, or tDCS alone in a blinded, parallel-design trial. Those randomized to an exercise intervention will receive individualized 30-min aerobic exercise prescriptions to achieve a moderate-intensity dosage, equivalent to the ventilatory anaerobic threshold determined by cardiopulmonary assessment, to sufficiently increase cortical excitability. The tDCS protocol consists of 20 min sessions at $2 \mathrm{~mA}, 5$ times per week for 2 weeks applied through $35 \mathrm{~cm}^{2}$ bitemporal electrodes. Our primary aim is to assess the efficacy of exercise primed tDCS for improving global cognition using the Montreal Cognitive Assessment (MoCA). Our secondary aims are to evaluate the efficacy of exercise primed tDCS for improving specific cognitive domains using various cognitive tests (n-back, Word Recall and Word Recognition Tasks from the Alzheimer's Disease Assessment Scale-Cognitive subscale) and neuropsychiatric symptoms (Neuropsychiatric Inventory). We will also explore whether exercise primed tDCS is associated with an increase in markers of neurogenesis, oxidative stress and angiogenesis, and if changes in these markers are correlated with cognitive improvement.
\end{abstract}

\footnotetext{
*Correspondence: krista.lanctot@sunnybrook.ca

${ }^{4}$ Department of Psychiatry, Division of Geriatric Psychiatry, Sunnybrook

Health Sciences Centre, 2075 Bayview Avenue, Toronto, Ontario M4N

3M5, Canada

Full list of author information is available at the end of the article
}

(C) The Author(s) 2021. Open Access This article is licensed under a Creative Commons Attribution 4.0 International License, which permits use, sharing, adaptation, distribution and reproduction in any medium or format, as long as you give appropriate credit to the original author(s) and the source, provide a link to the Creative Commons licence, and indicate if changes were made. The images or other third party material in this article are included in the article's Creative Commons licence, unless indicated otherwise in a credit line to the material. If material is not included in the article's Creative Commons licence and your intended use is not permitted by statutory regulation or exceeds the permitted use, you will need to obtain permission directly from the copyright holder. To view a copy of this licence, visit http://creativecommons.org/licenses/by/4.0/. The Creative Commons Public Domain Dedication waiver (http://creativeco mmons.org/publicdomain/zero/1.0/) applies to the data made available in this article, unless otherwise stated in a credit line to the data. 
Discussion: We describe a novel clinical trial to investigate the effects of exercise priming before tDCS in patients with $\mathrm{MCl}$ or mild AD. This proof-of-concept study may identify a previously unexplored, non-invasive, non-pharmacological combination intervention that improves cognitive symptoms in patients. Findings from this study may also identify potential mechanistic actions of tDCS in MCl and mild AD.

Trial registration: Clinicaltrials.gov, NCT03670615. Registered on September 13, 2018.

Keywords: Transcranial direct current stimulation, Exercise, Priming, Mild cognitive impairment, Alzheimer's disease, Neurogenesis, Angiogenesis, Cognition, Neuropsychiatric symptoms, Clinical trial

\section{Background}

Dementia is a neurocognitive disorder (NCD) characterized by a progressive deterioration of cognitive and functional abilities [1]. Alzheimer's Disease (AD) is the most common form of dementia, accounting for approximately $60-70 \%$ of all cases [2]. The diagnosis of a NCD, according to the Diagnostic and Statistical Manual of Mental Disorders -5th edition (DSM-5), can be classified as mild or major. Mild NCD, often referred to as Mild Cognitive Impairment (MCI), requires the presence of modest cognitive deficits without interference in everyday activities, whereas major NCD including $\mathrm{AD}$ requires both significant cognitive deficits and a reduced ability to perform everyday activities [3].

Cognitive problems are core deficits in MCI and AD. The earliest and most common clinical manifestation involves episodic memory impairment and a diminished ability to encode new material into long-term memory [4]. The temporal lobes of the brain are key structures in episodic memory, learning, and recall, and are damaged in $\mathrm{AD}$ [5]. These deficits are closely associated with neuronal hypoactivity and synaptic dysfunction [6-10].

Cholinesterase inhibitors (ChEIs), including donepezil, rivastigmine, and galantamine, as well as the N-methyl$\mathrm{D}$-aspartate antagonist, memantine, are approved for the symptomatic management of global cognitive impairment in $\mathrm{AD}$ [11]. Although those drugs improve cognitive performance, they are commonly associated with adverse events (AEs), such as drowsiness, loss of appetite, nausea, and vomiting [12]. Memantine, specifically, lacks evidence for efficacy in patients with mild AD [13]. There is also a lack of consensus among clinicians regarding issues related to initiation, optimal duration, and discontinuation [14]. The first disease modifying therapy, aducanumab, was recently approved by the FDA for AD, however, its approval is controversial due to ambiguous clinical trial results [15]. In patients with $\mathrm{MCI}$, evidence does not support the use of ChEIs or memantine [16, 17], underscoring the need for effective treatments in this population in order to treat cognitive impairment in the early stages and help delay the progression from MCI to $\mathrm{AD}$.
Individuals with $\mathrm{AD}$ frequently have neuropsychiatric symptoms (NPS). In a recent retrospective study, the most prevalent NPS among AD patients included apathy (51.9\%) followed by irritability $(41.0 \%)$ and depression (36.4\%) [18]. Sleep disturbances (31.7\%) were also commonly present in $\mathrm{AD}$ [18] and can have a negative impact on both quality of life and caregiver burden [19]. Current pharmacological treatments for NPS in dementia are only modestly effective and can be associated with significant adverse effects [20].

Transcranial direct current stimulation (tDCS) is a non-invasive brain stimulation technique that consists of applying a constant, low electric current between electrodes over the scalp in order to modulate cortical excitability [21]. Anodal tDCS, considered an excitatory stimulation, reduces the threshold required for neuronal firing and has been shown to improve neural efficiency, mood, and cognition in healthy as well as depressed and AD patients [22]. Similar to other types of brain stimulation, $\mathrm{tDCS}$ requires active neurons [23, 24], affecting the neurons that are closer to the discharge threshold (known as the activity-dependent model) [25, 26]. The effects of tDCS depend on the stimulation parameters used; however, studies have demonstrated that a single session of tDCS can induce changes in neuronal activity for up to $2 \mathrm{~h}$ [27]. tDCS has been used in numerous studies involving older, frail participants with no serious adverse events (SAEs) noted [28, 29]. In a review examining 158 studies, commonly reported side-effects included burning sensation, itching, tingling, headache, discomfort, dizziness, erythema, and fatigue. However, the risk of AEs did not increase in repeated sessions of active tDCS compared to sham tDCS [30].

To date, 21 studies have investigated the effects of repeated sessions of tDCS on cognitive performance in MCI and AD populations [31-51]. Some studies found that repeated sessions of tDCS significantly improved global cognition in mild AD patients [33, 52]. In addition, recall, recognition, and working memory have improved following single and repeated sessions of tDCS in mild $\mathrm{AD}[32,53-58]$. However, there was significant variability in treatment response which may be related to individual neurophysiological and biological status [59], including 
the level of cortical activity at the time of stimulations [60]. Based on new research on the activity-dependent model, studies have begun to investigate the use of combining other cognitive enhancing interventions with tDCS to prime neurons of interest [61]. In healthy participants, tDCS applied during a cognitive task resulted in greater improvement in performance of that cognitive task compared to when tDCS was applied at rest and when sham was applied during the task [62]. Although few studies have examined these combined therapies in AD patients, tDCS with cognitive training has been associated with greater improvements on some measures of cognition compared to sham tDCS with cognitive training, including the digit span, and trained and untrained picture-naming tasks [40].

Emerging evidence suggests that exercise may be an effective primer: a single bout of moderate-intensity aerobic exercise in healthy adults has been demonstrated to facilitate long-term potentiation-like neuroplasticity through increased motor-evoked potentials when exercise is performed prior to paired associative stimulation [27, 63]. Those findings were also associated with improved motor learning in participants who received exercise prior to stimulation compared to the rest condition (no exercise). In another study, a single bout of exercise (20 min of moderate-intensity cycling) resulted in increased cortical excitability and decreased intracortical inhibition compared to those who did not undergo an exercise session prior to stimulation [64]. Patients with Parkinson's disease who received repeated sessions of tDCS with physical therapy demonstrated greater improvements in measures of verbal fluency compared to those who received sham and physical therapy [65]. A direct combination of tDCS and aerobic exercise may provide synergistic effects where aerobic exercise provokes large-scale changes across the brain, priming the brain for focal modulation, and leading to more robust effects [27]. In our previous study, the subgroup of participants who had cognitive impairment (baseline MoCA score $\leq 25)$ and who exercised at at least moderateintensity level $\left(\geq 70 \%\right.$ of peak oxygen uptake $\left.\left(\mathrm{VO}_{2 \text { peak }}\right)\right)$ significantly improved on the MoCA at 3 months [66]. Those results suggest that moderate-intensity exercise can improve global cognitive function in individuals who are cognitively impaired, and is consistent with exercise being an effective primer to enhance the cognitive benefits of $\mathrm{tDCS}$.

In preclinical studies, $\mathrm{tDCS}$ has been shown to enhance secretion of brain-derived neurotrophic factor (BDNF), a neurotrophin with potent effects on neuronal survival and plasticity [67] and memory function [27, 68-71]. In vitro, application of direct current stimulation may also be associated with increases in angiogenic markers including vascular endothelial growth factor (VEGF) and angiopoietin-2 (ANGP-2) [72], which may play an important role in restoring memory through vascular survival [73]. In a vascular dementia mouse model, tDCS modulated oxidative stress through reduction of reactive oxygen species [74]. Reactive oxidative species can induce angiogenesis, and promote the production of angiogenic factors, such as VEGF [75]. These findings suggest that cognitive effects of tDCS may be partly mediated by neurogenesis and angiogenesis. In $\mathrm{AD}$, peripheral BDNF concentrations are lower compared to controls [76]. In addition, vascular abnormalities leading to hypoperfusion are associated with increases in anti-angiogenic markers such as endostatin, which can contribute to $\mathrm{AD}$ pathology [77]. Although studies have demonstrated improved cognitive performance following tDCS in patients with MCI or $\mathrm{AD}[27,60]$, there are no studies that have investigated angiogenic or neurogenic markers that may be mediating tDCS-induced cognitive improvement in $\mathrm{MCI}$ or $\mathrm{AD}$ patients. Given the negative impact of cognitive impairment in individuals with $\mathrm{MCI}$ and $\mathrm{AD}$, this parallel-design study aims to determine whether a combination therapy with tDCS and exercise priming effectively improves cognitive function and NPS, and whether cognitive response to tDCS treatment is associated with markers of neurogenesis and angiogenesis.

\section{Objectives}

The primary objective of the Exercise as a Primer for Excitatory Stimulation Study (EXPRESS) is to determine the efficacy of a combined exercise and tDCS intervention to enhance the effect of tDCS on global cognitive function.

Secondary objectives: to explore the efficacy of a combined exercise and tDCS intervention to enhance the effect of tDCS on specific cognitive domains and NPS.

Exploratory objectives: To identify mechanistic correlates of cognitive response to tDCS treatment through analysis of blood biomarkers.

\section{Methods \\ Study population and eligibility criteria}

Participants will be recruited through outpatient clinics within Sunnybrook Health Sciences Centre (SHSC) and referrals from physicians outside of SHSC within the Greater Toronto Area. Participants will be assessed based on inclusion and exclusion criteria highlighted in Table 1.

\section{Study design}

This is a randomized, blinded, repeated-session, paralleldesign study. Eligible participants will be randomized to one of three interventions: Exercise primer with tDCS, 
Table 1 List of inclusion and exclusion criteria

\begin{tabular}{|c|c|}
\hline Inclusion & Exclusion \\
\hline $\begin{array}{l}\text { - Males or females } \geq 50 \text { years of age (on day of randomization) } \\
\text { - Clinical diagnosis of major or mild neurocognitive disorder due to AD } \\
\text { or mixed AD/vascular disease following the DSM-5 [78] criteria by a } \\
\text { psychiatrist } \\
\text { - Mild severity of impairment (sMMSE score } \geq 19 \text { ) [79] } \\
\text { - Read and communicate in English }\end{array}$ & $\begin{array}{l}\text { - Change in cognitive-enhancing medications (ChEls and/or memantine) } \\
\text { less than } 3 \text { months prior to study screen } \\
\text { - Change in anticonvulsants or psychotropic medications less than } 1 \text { month } \\
\text { prior to study screen } \\
\text { - Currently taking benzodiazepines } \\
\text { - Presence of metal implants that would preclude safe use of tDCS (e.g. } \\
\text { pace-maker) } \\
\text { - Significant neurological condition (e.g., epilepsy, Parkinson's disease, } \\
\text { multiple sclerosis) } \\
\text { - Current psychiatric disorders (e.g. schizophrenia, bipolar disorder, depres- } \\
\text { sion, psychosis) or current substance abuse disorder } \\
\text { - Medical contraindication to increasing physical activity level according to } \\
\text { the Canadian Society of Exercise Physiology Questionnaire [80] }\end{array}$ \\
\hline
\end{tabular}

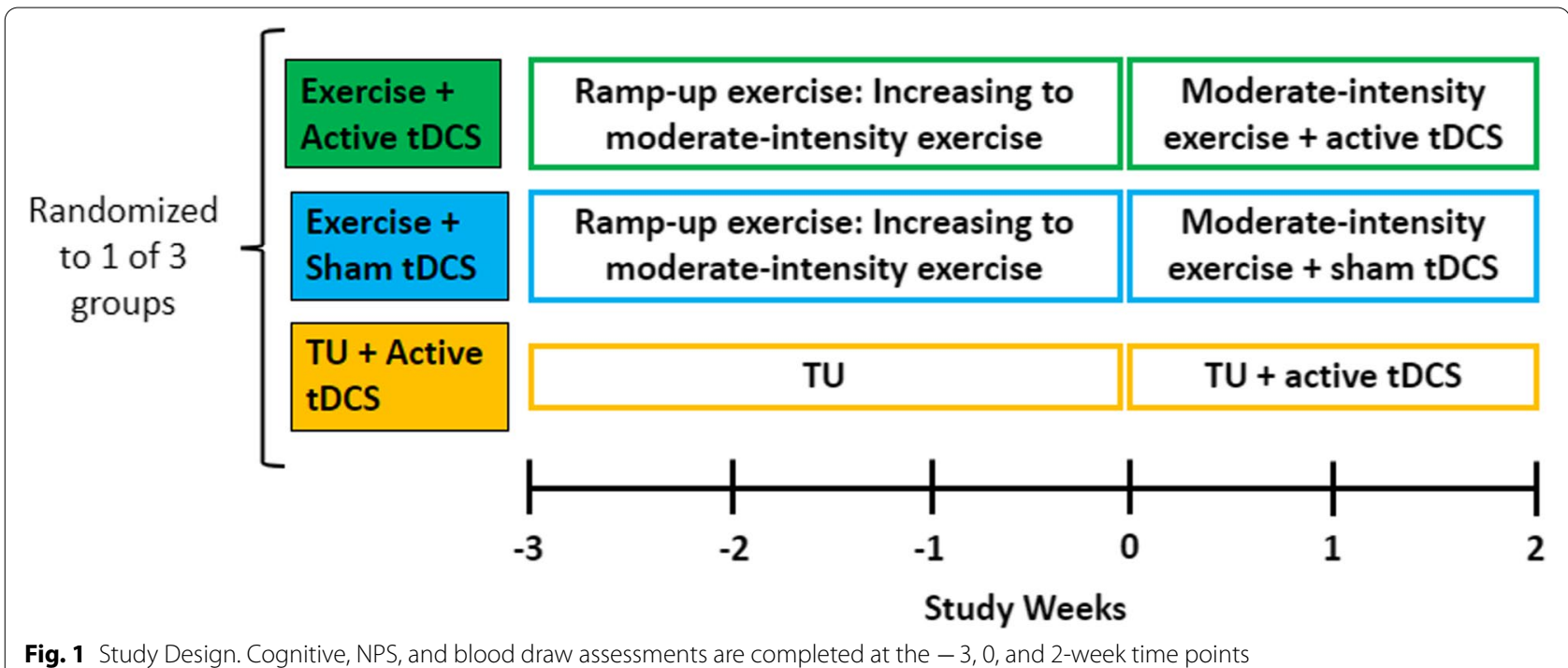

Treatment as usual (TU) with tDCS, or Exercise primer with sham tDCS (Fig. 1).

Assessments will be conducted at the following time points: Screening/Baseline (T1): within two weeks of beginning the 5-week study and/or on the first day of study initiation prior to any exercise intervention/TU, Midpoint (T2): 3 weeks into the study and prior to tDCS/ sham, Endpoint (T3): study completion/at the end of the 5-week study, and Follow-Up with a physician (T4) (Table 2). At screening, inclusion and exclusion criteria (Table 1) will be assessed and demographic information (e.g. age, sex, ethnicity, education, sleep, caffeine, and alcohol intake, concomitant medications, comorbid illnesses, surgical and medical history, smoking status, as well as physical fitness level) and standardized MiniMental State Examination (sMMSE) score will be collected. At baseline, midpoint, and endpoint, cognitive and NPS outcomes, as well as blood biomarkers, will be collected. For participants randomized to receive an exercise intervention, an individual exercise prescription will be administered at baseline. For participants randomized to TU, written information in accordance with the Canadian Physical Activity Guidelines for older adults will be given at baseline. Exercise will be documented weekly using the Leisure-Time Exercise Questionnaire (LTEQ) for all participants to monitor exercise frequency and intensity throughout the study. Participants will be followed up by a qualified investigator (QI) or designated qualified physician within 2 weeks of study endpoint or termination.

\section{Interventions}

Exercise intervention

Current guidelines recommend that older adults participate in at least $150 \mathrm{~min} /$ week of moderate-intensity aerobic activity [81], a level sufficient to increase cortical 
Table 2 Schedule of assessments

\begin{tabular}{|c|c|c|c|c|}
\hline Visit & $\begin{array}{l}\text { Screening/ Baseline } \\
\text { T1 } \\
\text { Study initiation }\end{array}$ & $\begin{array}{l}\text { Midpoint } \\
\text { T2 } \\
\text { 3-weeks }\end{array}$ & $\begin{array}{l}\text { Endpoint }^{\mathrm{a}} \\
\text { T3 } \\
\text { 5-weeks } \\
\text { / study } \\
\text { endpoint }\end{array}$ & $\begin{array}{l}\text { Follow-up } \\
\text { T4 } \\
\text { Within } 2 \text { weeks of T3 }\end{array}$ \\
\hline \multicolumn{5}{|l|}{ Demographics } \\
\hline $\begin{array}{l}\text { Clinical diagnosis of mild or major NCD due to AD or mixed AD/vascular } \\
\text { disease following the DSM-5 criteria by a psychiatrist }\end{array}$ & $x$ & & & \\
\hline Demographics & $x$ & & & \\
\hline Surgical and health history & $x$ & & & \\
\hline Comorbid IIInesses and concomitant medications & $x$ & $x$ & $x$ & \\
\hline Sleep, caffeine, alcohol intake, smoking status & $x$ & $x$ & $x$ & \\
\hline Standardized mini-mental state examination & $x$ & & & \\
\hline $\begin{array}{l}\text { Canadian society of exercise physiology physical activity readiness } \\
\text { questionnaire-plus }\end{array}$ & $x$ & & & \\
\hline \multicolumn{5}{|l|}{ Outcomes } \\
\hline Montreal cognitive assessment & $x$ & $x$ & $x$ & \\
\hline Word recognition task & $x$ & $x$ & $x$ & \\
\hline Word recall task & $x$ & $x$ & $x$ & \\
\hline n-back & $x$ & $x$ & $x$ & \\
\hline Neuropsychiatric inventory & $x$ & $x$ & $x$ & \\
\hline Blood biomarkers & $x$ & $x$ & $x$ & \\
\hline \multicolumn{5}{|l|}{ Safety } \\
\hline Adverse Events & $x$ & $x$ & $x$ & \\
\hline \multicolumn{5}{|l|}{ Other } \\
\hline Leisure-time exercise questionnaire ${ }^{b}$ & $x$ & $x$ & $x$ & \\
\hline $\begin{array}{l}\text { Follow-up assessment with qualified investigator or designated qualified } \\
\text { physician }\end{array}$ & & & & $x$ \\
\hline
\end{tabular}

excitability [64]. Following an initial cardiopulmonary exercise test at University Health Network-Toronto Rehabilitation Institute (UHN-TRI) to assess baseline cardiorespiratory fitness, an initial walking prescription will be given and set at a distance of approximately $0.8-1.6 \mathrm{~km}$ for $10-30 \mathrm{~min}$ depending on participant tolerance. The intensity will be at the ventilatory anaerobic threshold (VAT) and/or $60-80 \%$ of $\mathrm{VO}_{2 \text { peak }}$. Prescriptions will be progressed every 3-4 sessions for 3 weeks to increase distance to reach a duration of $30 \mathrm{~min}$ and intensity to the level of the VAT and/or $80 \%$ of $\mathrm{VO}_{2 \text { peak. }}$. Participants will be instructed to exercise at UHN-TRI 3 times per week and at home 2 times per week for the first 3 weeks while the exercise intensity and/or duration is being increased. During the last 2 weeks, participants will be instructed to exercise at UHN-TRI 5 times per week. All exercise at UHN-TRI will be supervised and heart rate will be monitored using Polar heart rate monitors to ensure moderate intensity exercise is maintained. Prescribing intensity based on the VAT provides a metabolically uniform moderate intensity exercise prescription. This is especially important as not all individuals are able to reach a "true" physiological maximum on the exercise stress test. Our research group previously conducted a study in participants undergoing a 6-month cardiac rehabilitation exercise program at UHN-TRI, and demonstrated that exercise in older adults is a feasible intervention with $85.6 \%$ adherence [66].

\section{Treatment as usual (TU)}

TU will include routine advice about physical activity for older adults and written information in accordance with the Canadian Physical Activity Guidelines for older adults [82] as is standard of care. Physical activity in older adults often remains below the recommended levels of at least $150 \mathrm{~min}$ per week [83]. Participants in this group will not receive a personalized exercise plan and will not have contact from an exercise physiologist during the intervention. Participants will be instructed not to exercise before stimulation. 

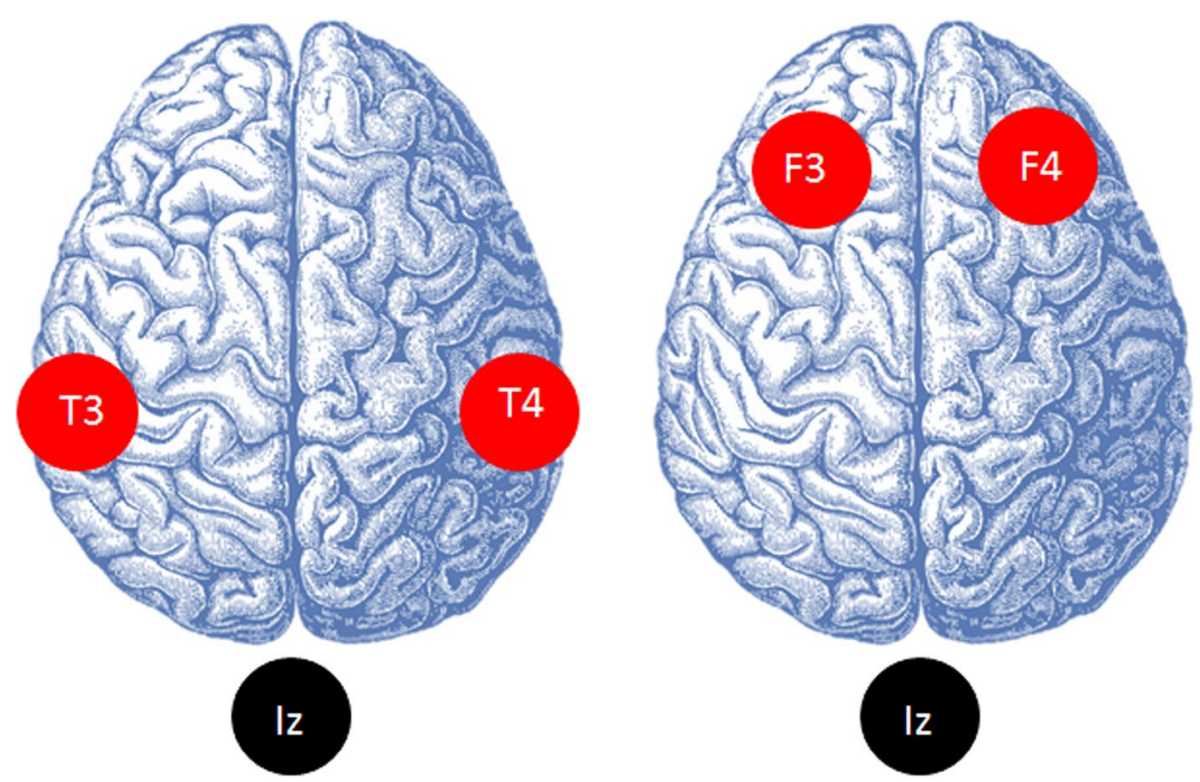

Fig. 2 Schematic of electrode placements. Red and black circles represent anodal and return electrodes respectively

\section{tDCS}

tDCS will be delivered by an Eldith DC stimulator (Magstim Company Ltd. UK), using three saline-soaked sponge electrodes held in place by an elastic band cap. The two anodal electrodes will be placed bitemporally over the left and right medial temporal lobes (T3+T4 hemispheres) according to the International 10-20 system of electrode placement. Bitemporal placement with tDCS has shown cognitive improvements in MCI and AD [58], and neuroimaging findings on neuronal loss or hypoactivity in temporal regions of both hemispheres [84] also support the regions' activity in memory processing. The return electrode will be placed at the inion (Iz) (Fig. 2). In each session, tDCS would be administered with a current strength of $2 \mathrm{~mA}$ for $20 \mathrm{~min}$ as these parameters are safe and commonly used in clinical research [85]. All study participants randomized to tDCS will receive tDCS 5 times/week for 2 weeks. Those randomized to receive exercise will be given tDCS following their exercise sessions. Compliance, including exercise and tDCS frequency, will be measured and documented.

\section{Sham tDCS}

The same procedure for tDCS will be used for the sham condition, except the $2 \mathrm{~mA}$ current will only be applied in the ramp-up phase (first $30 \mathrm{~s}$ ). In previous studies [28, 86], this approach has ensured that the participants are blind to the type of stimulation (active versus sham).

\section{Study outcomes \\ Cognitive assessments}

We will administer a comprehensive cognitive battery composed of neuropsychological tests that have been previously used in mild AD, and have demonstrated sensitivity to cognitive changes following tDCS [87]. Alternative versions will be used to avoid learning effects where applicable. The MoCA [88], a brief test of global cognition and a sensitive validated tool for detecting early cognitive changes in mild $\operatorname{AD}[89,90]$, will be the primary outcome measure. The MoCA assesses multiple domains including orientation, attention, executive function, language and abstraction in addition to memory [90]. Two weeks is appropriate to observe changes in global cognition [33] following a cognitive enhancement intervention.

Working memory will be assessed using the n-back [91]. The Word Recognition Task and the Word Recall Tasks from the Alzheimer's Disease Assessment ScaleCog (ADAS-Cog) [92] will be used to assess recognition memory and recall memory respectively. These domains have also been shown to improve with exercise interventions [93, 94]. The sMMSE [79] will be administered at screening to assess for severity of cognitive impairment; it is the most common short screening tool for assessing cognitive impairment overall [95].

\section{NPS}

The Neuropsychiatric Inventory (NPI) [96] will be used to measure apathy, agitation, delusions, hallucinations, depression, euphoria, aberrant motor behaviour, 
irritability, disinhibition, anxiety, sleeping, and appetite disturbances in patients and to evaluate caregiver distress. Each domain is scored for frequency, severity and caregiver distress. Doman score is obtained by multiplying frequency and severity. Total NPI score is obtained by summing the individual domain scores. The NPI has good content validity, internal consistency, testretest and interrater reliability, and is commonly used in research for the assessment of NPS in mild AD [97]. It will be administered to the patient's caregiver through a standardized interview. Studies have shown that decreased cognition in MCI and AD is highly associated with increased NPS [98-100]. Therefore, effective treatments for cognitive impairment may also improve NPS in patients with AD.

\section{Physical activity}

The LTEQ [87] will be administered to the patient or the patient's caregiver weekly to measure frequency and intensity of exercise completed. The Canadian Society of Exercise Physiology Questionnaire [80] will be administered to the patient or the patient's caregiver at screening to assess for medical contraindications to a physical activity.

\section{Biomarker assays}

Angiogenic and neurogenic markers associated with exercise and tDCS outcomes, including BDNF, VEGF, ANGP-2, and endostatin, will be analysed using enzymelinked immunosorbent assay [76, 101-103]. Samples for analyses will be frozen $\left(-80^{\circ} \mathrm{C}\right)$ immediately and batched for analysis. These angiogenesis and neurogenic markers have previously been shown to change following two or more weeks of an exercise intervention [104-108].

\section{Randomization and blinding}

A block randomization code will be computer-generated at SHSC and remain locked in a secure location in the department. The research personnel who will be administering the cognitive assessments will not be the interventionist administering the tDCS/sham intervention, and, with the remaining team members will be blinded to treatment allocation and block size until the final patient has completed follow-up and the database is locked. The patients will also be blinded to stimulation type. Unblinding will not be allowed unless there exist exceptional clinical circumstances that justify it (i.e., necessary for acute medical management of SAEs) and only after approval by the principal investigator (PI) or a QI.

\section{Safety profile and monitoring}

Side-effects will be monitored using a customized checklist after each tDCS session. All emerging AEs that are clinically relevant based on a QI or designated qualified physician's assessment will be noted and monitored until resolution. AEs will be appropriately described, i.e.: the association with the intervention will be coded as not related, possibly related or related; the determination of the severity and association will be decided by a QI. The QIs for this study will also be acting as the safety monitor, viewing all AEs.

New medical conditions developed during the study including musculoskeletal, cardiovascular or neurological symptoms which may make continued exercise unsafe will be reported to a QI or the designated qualified physician. Patients will be evaluated by a study physician to ensure that the participant received appropriate medical attention and to determine whether the participant may safely continue or discontinue the study.

An SAE is any untoward medical occurrence that results in death, is life-threatening (defined as an event in which the patient was at risk of death at the time of the event; it does not refer to an event which hypothetically might have caused death if it were more severe), results in persistent or significant disability or incapacity, requires hospitalization or causes prolongation of existing hospitalization, results in the development of drug dependency or drug abuse or is an important medical event defined as a medical event(s) that may not be immediately life-threatening or result in death or hospitalization but, based upon appropriate medical and scientific judgment, may jeopardize the patient or may require intervention (e.g., medical, surgical) to prevent one of the other serious outcomes listed in the definition above. SAEs will be collected during the study and will be followed until event resolution or stabilization. All SAEs that are unexpected and potentially related to the research will be reported in an expedited manner to the Research Ethics Board at SHSC.

\section{Adherence}

Administration of exercise will be supervised by a physician, exercise physiologist, and designated research staff. Exercise frequency and intensity will be measured by the LTEQ weekly. Adherence to exercise regimen will be measured by information from the LTEQ and included as a covariate in statistical analyses.

\section{Statistical plan Sample size calculation}

The sample size calculation was performed using G*Power version 3.1.9.2 (Kiel, Germany). The sample size was based on findings from a study reporting a 2-point change in MMSE score following anodal tDCS in mild to moderate AD [33]. No studies to date have assessed changes in the MoCA following anodal tDCS. However, 
a strong correlation between the MMSE and MoCA $(\mathrm{r}=0.86)$ in mild AD [89] implies that a similar change in the MoCA could be found in this population. Based on that assumption, a sample size of 60 completers $(20$ per treatment group) achieves $83 \%$ power for a repeated measures analysis of covariance (ANCOVA), adjusting for 4 covariates with an alpha of 0.05 to detect a medium effect size.

\section{Statistical analyses}

Multiple imputation methods will be used prior to analysis if missing data exceed $10 \%$. A repeated measures ANCOVA will be used to assess between group differences in change in MoCA score over 2 weeks (treatment group $\mathrm{x}$ time interaction). Age and education will be added as covariates. A treatment group $\mathrm{x}$ time interaction will also be used to explore between-group differences in the n-back, ADAS-Cog, total NPI score, and NPI subscores. Repeated measures ANCOVAs will also be used to assess differences in markers of interest between treatment groups. Associations between changes in concentrations of BDNF, VEGF, ANGP-2, endostatin and MoCA scores over 2 weeks will be assessed using repeated measures linear regressions. It is expected that randomization will balance possible confounders.

\section{Data collection and management}

Data will be collected on printed source documents/case report forms (CRFs). Source documents/CRFs include demographic information, medical information, cognitive as well as mood and behaviour assessments. Source documents, CRFs, and other records pertaining to the conduct of this study will be retained for 10 years, as per SHSC Research Ethics Board (REB) recommendations.

\section{Discussion}

Recent studies have demonstrated that, individually, exercise and tDCS can enhance cortical excitability and improve cognitive functions in $\mathrm{MCI}$ and $\mathrm{AD}$ populations $[60,109]$. Since tDCS may be most effective when applied to active neurons $[23,24]$, the combined treatment strategy, exercise-primed tDCS, may have greater efficacy to improve cognition in MCI and AD patients. To our knowledge, no studies have examined the cognitive response of exercise-primed tDCS in MCI or AD. The findings from this trial may provide a novel combined treatment strategy for $\mathrm{MCI}$ and mild $\mathrm{AD}$ patients.

A small number of studies have examined other combined interventions in $\mathrm{MCI}$ and $\mathrm{AD}$, such as $\mathrm{CT}$ and tDCS [35, 40, 43, 44]; those studies only showed improvements in specific targeted cognitive domains, and it still remains unclear whether the combined treatment produces better outcomes than tDCS alone [110]. Exercise can induce large-scale changes across the brain, priming the brain for focal modulation, and leading to more robust effects with tDCS [27]. Also, exercise-primed tDCS is advantageous over other multimodal strategies, including exercise and transcranial magnetic stimulation, because it is easy to implement, time and cost-efficient, and safe with no-known severe side effects [27]. Recently, tDCS has been investigated as a home-based therapy for MCI patients [111].

Neurogenic factors, such as BDNF, have been suggested to have an influence on the response to tDCS [112]. Evidence has demonstrated that tDCS regulates BDNF expression and overall genetic variances of BDNF expression may be involved in the differences of individual responses to tDCS stimulation [71, 112]. Currently, there have been no studies in $\mathrm{MCI}$ or $\mathrm{AD}$ patients that have investigated neurogenic mechanisms of tDCS response. Findings on potential blood biomarkers associated with exercise, tDCS outcomes, angiogenesis and neurogenesis from this study may also identify potential mechanisms to delay impairment and preserve cognition in MCI and mild AD patients.

Given the potential wide availability of tDCS (available for home use) and current knowledge supporting efficacious exercise interventions, combined with the low risk of both interventions, this combination is highly feasible for widespread early intervention. As the populations suffering from $\mathrm{MCI}$ and $\mathrm{AD}$ are largely elderly individuals who are heavily burdened by pharmacological agents, the ability to introduce a non-pharmacological treatment is of great importance and value to patients and clinicians.

\footnotetext{
Abbreviations

AD: Alzheimer's Disease; ADAS-Cog: Alzheimer's Disease Assessment ScaleCog; AEs: Adverse Events; ANCOVA: Analysis of Covariance; ANGP-2: Angiopoietin-2; BDNF: Brain-derived Neurotrophic Factor; ChEls: Cholinesterase Inhibitors; CRFs: Case Report Forms; DSM-5: Diagnostic and Statistical Manual of Mental Disorders ; EXPRESS: Exercise as a Primer for Excitatory Stimulation Study; Iz: Inion; LTEQ: Leisure-Time Exercise Questionnaire; MCl: Mild Cognitive Impairment; MoCA: Montreal Cognitive Assessment; NCD: Neurocognitive Disorder; NPI: Neuropsychiatric Inventory; NPS: Neuropsychiatric Symptoms; PI: Principal Investigator; QI: Qualified Investigator; REB: Research Ethics Board; SAEs: Serious Adverse Events; SHSC: Sunnybrook Health Sciences Centre; sMMSE: Standard Mini-Mental State Examination; TU:Treatment As Usual; tDCS: Transcranial Direct Current Stimulation; UHN-TRI: University Health Network-Toronto Rehabilitation Institute; VAT: Ventilatory Anaerobic Threshold; VEGF: Vascular Endothelial Growth Factor; $V_{2}$ aeak: Peak Oxygen Uptake.
}

\section{Acknowledgements}

This work was previously presented at the 3rd International Brain Stimulation Conference:

Liu C, Herrmann N, Gallagher D, Rajji T, Oh P, Marzolini S, Banks L, Vieira D, Lanctot K. Designing a randomized, sham-controlled, parallel-design trial to investigate the effects of a combined exercise priming and transcranial direct current stimulation intervention in mild cognitive impairment and alzheimer's disease. March-April 2019 Brain Stimulation 12(2): 425. https://doi.org/10. 1016/j.brs.2018.12.378 
The authors would like to thank the patients, families and medical staff at Sunnybrook Health Sciences Centre and Toronto Rehabilitation Institute for participation in this trial.

\section{Trial status}

Patient recruitment is currently ongoing.

\section{Trial sponsor}

Sunnybrook Research Institute, Toronto, Ontario, M4N3M5. They have no influence on data analyses or writing of the manuscript.

\section{Authors' contributions}

$\mathrm{KLL}, \mathrm{CSL}$ and NH designed the study. KLL, NH, DG, PIO, SM, TKR, ACA, AK, PP, JC and CSL contributed to the protocol development. The Neuropsychopharmacology Research Group, (KLL, DG, NH, MJR, DV, CSL, BS), will be primarily responsible for recruitment, assessments, data management, analysis interpretation of data, and publication of results. CSL, KLL, NH, BS, and JB are major contributors in writing the manuscript. All authors read and approved the final manuscript.

\section{Funding}

This project has received funding from the Canadian Institutes of Health Research Priority Announcements for Neurosciences, Mental Health and Addiction. The funding body had no role in the design of the study and collection, analysis, and interpretation of data and in writing the manuscript.

\section{Availability of data and materials}

The datasets generated during and/or analysed for the proposed study will not be publicly available as institutional approval would be required for data sharing. However, following publication of peer-reviewed journal articles, congregated data will be available from the corresponding author upon request.

\section{Declarations}

\section{Ethics approval and consent to participate}

Ethics approval for this trial (protocol version 1.3, July 31, 2020) was obtained from Sunnybrook's REB (075-2018) and University Health Network REB (185508). These research ethics boards will approve all subsequent amendments to the protocol. If participants did not have the cognitive capacity to consent, their appointed powers of attorney provide written informed consent before their participation in the trial. Participants will be informed of any changes or new information that may affect their safety and willingness to continue participating in the study.

\section{Consent for publication}

Not applicable.

\section{Competing interests}

A list of investigators with competing interests and the details they have declared is as follows:KLL has received research grants from the National Institutes of Aging, Alzheimer's Drug Discovery Foundation, the Alzheimer Society of Canada, Alzheimer's Association, Canadian Institutes of Health Research, Weston Brain Institute; honoraria from BioXcel, Cerevel, Eisai and Praxis. This research is supported by the Canadian Institutes of Health Research Priority Announcement for Neurosciences, Mental Health and Addiction. $\mathrm{NH}$ has received peer-reviewed research grants from the Alzheimer Drug Discovery Foundation, the Alzheimer Society of Canada, the National Institute of Health, Canadian Institutes of Health Research.

The remaining authors have no competing interests to declare.

\section{Author details}

'Department of Pharmacology \& Toxicology, University of Toronto, 1 King's College Circle, Toronto, ON M5S 3K1, Canada. ${ }^{2}$ Neuropsychopharmacology Research Group, Hurvitz Brain Sciences Program, Sunnybrook Research Institute, 2075 Bayview Avenue, Toronto, ON M4N 3M5, Canada. ${ }^{3}$ Department of Psychiatry, University of Toronto, Toronto, ON, Canada. ${ }^{4}$ Department of Psychiatry, Division of Geriatric Psychiatry, Sunnybrook Health Sciences Centre, 2075 Bayview Avenue, Toronto, Ontario M4N 3M5, Canada. ${ }^{5}$ Department of Biology, Faculty of Science, The University of Western Ontario, London, ON, Canada. ${ }^{6}$ Cardiovascular Prevention and Rehabilitation Program, KITE - Toronto
Rehabilitation Institute, University Health Network, 347 Rumsey Road, Toronto, ON M5G 1R7, Canada. ${ }^{7}$ Adult Neurodevelopment and Geriatric Psychiatry Division, Centre for Addiction \& Mental Health, 80 Workman Way, Toronto, ON M6J 1H4, Canada. ${ }^{8}$ Toronto Dementia Research Alliance, University of Toronto, Toronto, ON, Canada. ${ }^{9}$ Family \& Community Medicine, Sunnybrook Health Sciences Centre, 2075 Bayview Avenue, Toronto, ON M4N 3M5, Canada.

${ }^{10}$ Institute for Clinical Evaluative Sciences, Sunnybrook Health Sciences Centre, Toronto, ON, Canada.

Received: 31 August 2021 Accepted: 16 November 2021

Published online: 04 December 2021

\section{References}

1. 2015 Alzheimer's disease facts and figures. Alzheimers Dementia. 2015;11(3):332-384.

2. Organization WH. Dementia: key facts 2020 [updated 21 Sept 2020.

3. Association AP. Diagnostic and statistical manual of mental disorders, 5th edn 2013.

4. Karantzoulis S, Galvin JE. Distinguishing Alzheimer's disease from other major forms of dementia. Expert Rev Neurother. 2011;11(11):1579-91.

5. Braak H, Braak E. Evolution of neuronal changes in the course of Alzheimer's disease. J Neural Transm Suppl. 1998:53:127-40.

6. La Joie R, Perrotin A, Barre L, Hommet C, Mezenge F, Ibazizene M, et al. Region-specific hierarchy between atrophy, hypometabolism, and beta-amyloid (Abeta) load in Alzheimer's disease dementia. J Neurosci. 2012;32(46):16265-73.

7. Bejanin A, Schonhaut DR, La Joie R, Kramer JH, Baker SL, Sosa N, et al. Tau pathology and neurodegeneration contribute to cognitive impairment in Alzheimer's disease. Brain. 2017;140(12):3286-300.

8. Cavedo E, Pievani M, Boccardi M, Galluzzi S, Bocchetta M, Bonetti M, et al. Medial temporal atrophy in early and late-onset Alzheimer's disease. Neurobiol Aging. 2014;35(9):2004-12.

9. Reddy PH, Mani G, Park BS, Jacques J, Murdoch G, Whetsell W Jr, et al. Differential loss of synaptic proteins in Alzheimer's disease: implications for synaptic dysfunction. J Alzheimers Dis. 2005;7(2):103-17 discussion 73-80.

10. Wang HW, Pasternak JF, Kuo H, Ristic H, Lambert MP, Chromy B, et al. Soluble oligomers of beta amyloid (1-42) inhibit long-term potentiation but not long-term depression in rat dentate gyrus. Brain Res. 2002;924(2):133-40.

11. Campos C, Rocha NB, Vieira RT, Rocha SA, Telles-Correia D, Paes F, et al. Treatment of cognitive deficits in Alzheimer's disease: a psychopharmacological review. Psychiatr Danub. 2016;28(1):2-12.

12. Kobayashi H, Ohnishi T, Nakagawa R, Yoshizawa K. The comparative efficacy and safety of cholinesterase inhibitors in patients with mild-tomoderate Alzheimer's disease: a Bayesian network meta-analysis. Int J Geriatr Psychiatry. 2016;31 (8):892-904.

13. McShane R, Westby MJ, Roberts E, Minakaran N, Schneider L, Farrimond $L E$, et al. Memantine for dementia. Cochrane Database Syst Rev. 2019;(3). https://doi.org/10.1002/14651858.CD003154.pub6.

14. Deardorff WJ, Feen E, Grossberg GT. The use of cholinesterase inhibitors across all stages of Alzheimer's disease. Drugs Aging. 2015;32(7):537-47.

15. Servick K. Alzheimer's drug approved despite murky results. Science. 2021;372(6547):1141.

16. Cooper C, Li R, Lyketsos C, Livingston G. Treatment for mild cognitive impairment: systematic review. Br J Psychiatry. 2013;203(3):255-64.

17. J-y H, Besser LM, Xiong C, Kukull WA, Morris JC. Cholinesterase inhibitors may not benefit mild cognitive impairment and mild Alzheimer disease dementia. Alzheimer Dis Assoc Disord. 2019;33(2):87-94.

18. Anor CJ, O'Connor S, Saund A, Tang-Wai DF, Keren R, Tartaglia MC. Neuropsychiatric symptoms in Alzheimer disease, vascular dementia, and mixed dementia. Neurodegener Dis. 2017;17(4-5):127-34.

19. Khoo SA, Chen TY, Ang YH, Yap P. The impact of neuropsychiatric symptoms on caregiver distress and quality of life in persons with dementia in an Asian tertiary hospital memory clinic. Int Psychogeriatr. 2013;25(12):1991-9.

20. Phan SV, Osae S, Morgan JC, Inyang M, Fagan SC. Neuropsychiatric symptoms in dementia: considerations for pharmacotherapy in the USA. Drugs R D. 2019;19(2):93-115. 
21. Nitsche MA, Paulus W. Excitability changes induced in the human motor cortex by weak transcranial direct current stimulation. J Physiol. 2000;527:633-9.

22. Turi Z, Paulus W, Antal A. Functional neuroimaging and transcranial electrical stimulation. Clin EEG Neurosci. 2012;43(3):200-8.

23. Bikson M, Name A, Rahman A. Origins of specificity during tDCS: anatomical, activity-selective, and input-bias mechanisms. Front Hum Neurosci. 2013;7:688.

24. Dayan E, Censor N, Buch ER, Sandrini M, Cohen LG. Noninvasive brain stimulation: from physiology to network dynamics and back. Nat Neurosci. 2013;16(7):838-44.

25. Fertonani A, Miniussi C. Transcranial electrical stimulation: what we know and do not know about mechanisms. Neuroscientist. 2017;23(2):109-23.

26. Siebner HR, Hartwigsen G, Kassuba T, Rothwell JC. How does transcranial magnetic stimulation modify neuronal activity in the brain? Implications for studies of cognition. Cortex. 2009;45(9):1035-42.

27. Steinberg F, Pixa NH, Fregni F. A review of acute aerobic exercise and Transcranial direct current stimulation effects on cognitive functions and their potential synergies. Front Hum Neurosci. 2019;12:534.

28. Nitsche MA, Liebetanz D, Lang N, Antal A, Tergau F, Paulus W. Safety criteria for transcranial direct current stimulation (tDCS) in humans. Clin Neurophysiol. 2003;114(11):2220-2 author reply 2-3.

29. Bikson M, Grossman P, Thomas C, Zannou AL, Jiang J, Adnan T, et al. Safety of transcranial direct current stimulation: evidence based update 2016. Brain Stimul. 2016;9(5):641-61.

30. Nikolin S, Huggins C, Martin D, Alonzo A, Loo CK. Safety of repeated sessions of transcranial direct current stimulation: a systematic review. Brain stimul. 2018;11(2):278-88.

31. Cruz Gonzalez P, Fong KNK, Brown T. The effects of transcranial direct current stimulation on the cognitive functions in older adults with mild cognitive impairment: a pilot study. Behav Neurol. 2018;2018:5971385.

32. Boggio PS, Ferrucci $R$, Mameli F, Martins D, Martins $O$, Vergari $M$, et al. Prolonged visual memory enhancement after direct current stimulation in Alzheimer's disease. Brain Stimul. 2012:5(3):223-30.

33. Khedr EM, Gamal NF, El-Fetoh NA, Khalifa H, Ahmed EM, Ali AM, et al. A double-blind randomized clinical trial on the efficacy of cortical direct current stimulation for the treatment of Alzheimer's disease. Front Aging Neurosci. 2014;6:275.

34. Khedr EM, Salama RH, Abdel Hameed M, Abo Elfetoh N, Seif P. Therapeutic role of transcranial direct current stimulation in Alzheimer disease patients: double-blind, placebo-controlled clinical trial. Neurorehabil Neural Repair. 2019;33(5):384-94.

35. Cotelli M, Manenti R, Brambilla M, Petesi M, Rosini S, Ferrari C, et al. Anodal tDCS during face-name associations memory training in Alzheimer's patients. Front Aging Neurosci. 2014;6:38.

36. Suemoto CK, Apolinario D, Nakamura-Palacios EM, Lopes L, Leite REP, Sales MC, et al. Effects of a non-focal plasticity protocol on apathy in moderate Alzheimer's disease: a randomized, double-blind, shamcontrolled trial. Brain Stimul. 2014;7(2):308-13.

37. Penolazzi B, Bergamaschi S, Pastore M, Villani D, Sartori G, Mondini S. Transcranial direct current stimulation and cognitive training in the rehabilitation of Alzheimer disease: a case study. Neuropsychol Rehabil. 2015;25(6):799-817.

38. Bystad M, Rasmussen ID, Grønli O, Aslaksen PM. Can 8 months of daily tDCS application slow the cognitive decline in Alzheimer's disease? A case study. Neurocase. 2017;23(2):146-8.

39. Bystad M, Grønli O, Rasmussen ID, Gundersen N, Nordvang L, WangIversen $\mathrm{H}$, et al. Transcranial direct current stimulation as a memory enhancer in patients with Alzheimer's disease: a randomized, placebocontrolled trial. Alzheimers Res Ther. 2016;8:1-7.

40. Roncero C, Kniefel H, Service E, Thiel A, Probst S, Chertkow H. Inferior parietal transcranial direct current stimulation with training improves cognition in anomic Alzheimer's disease and frontotemporal dementia. Alzheimers Dement. 2017;3(2):247-53.

41. Yun K, Song I-U, Chung Y-A. Changes in cerebral glucose metabolism after 3 weeks of noninvasive electrical stimulation of mild cognitive impairment patients. Alzheimers Res Ther. 2016;8(1):49.

42. Im JJ, Jeong H, Bikson M, Woods AJ, Unal G, Oh JK, et al. Effects of 6-month at-home transcranial direct current stimulation on cognition and cerebral glucose metabolism in Alzheimer's disease. Brain Stimul. 2019;12(5):1222-8.

43. Das N, Spence JS, Aslan S, Vanneste S, Mudar R, Rackley A, et al. Cognitive training and transcranial direct current stimulation in mild cognitive impairment: a randomized pilot trial. Front Neurosci. 2019;13:307.

44. Costa V, Brighina F, Piccoli T, Realmuto S, Fierro B. Anodal transcranial direct current stimulation over the right hemisphere improves auditory comprehension in a case of dementia. NeuroRehabilitation. 2017;41(2):567-75

45. Gangemi A, Colombo B, Fabio RA. Effects of short- and long-term neurostimulation (tDCS) on Alzheimer's disease patients: two randomized studies. Aging Clin Exp Res. 2021;33(2):383-90.

46. Masse C, Chopard G, Magnin E, Nicolier M, Monnin J, Bennabi D, et al. Improvement of response to cueing after left prefrontal transcranial direct current stimulation in Alzheimers disease. 2018;8(5):1461-4.

47. Fileccia E, Di Stasi V, Poda R, Rizzo G, Stanzani-Maserati M, Oppi F, et al. Effects on cognition of 20-day anodal transcranial direct current stimulation over the left dorsolateral prefrontal cortex in patients affected by mild cognitive impairment: a case-control study. Neurol Sci. 2019:40(9):1865-72.

48. Gomes MA, Akiba HT, Gomes JS, Trevizol AP, de Lacerda ALT, Dias ÁM. Transcranial direct current stimulation (tDCS) in elderly with mild cognitive impairment: a pilot study. Dement Neuropsychol. 2019;13(2):187-95.

49. Inagawa T, Yokoi Y, Narita Z, Maruo K, Okazaki M, Nakagome K. Safety and feasibility of transcranial direct current stimulation for cognitive rehabilitation in patients with mild or major neurocognitive disorders: a randomized sham-controlled pilot study. Front Hum Neurosci. 2019;13:273.

50. Lu H, Chan SSM, Chan WC, Lin C, Cheng CPW, Linda Chiu Wa L. Randomized controlled trial of TDCS on cognition in 201 seniors with mild neurocognitive disorder. Ann Clin Translat Neurol. 2019;6(10):1938-48.

51. de Almeida Rodrigues ET, da Silva Machado DG, Leon de Mendonca CTP, da Rocha SC, Ries A, Torro N, et al. Multisite transcranial direct current stimulation in two patients with Alzheimer's disease: a 10-month follow-up study. Neurophysiol Clin. 2020;50(5):393-5.

52. Cai M, Guo Z, Xing G, Peng H, Zhou L, Chen H, et al. Transcranial direct current stimulation improves cognitive function in mild to moderate Alzheimer disease: a meta-analysis. Alzheimer Dis Assoc Disord. 2019;33(2):170-8.

53. Ferrucci R, Mameli F, Guidi I, Mrakic-Sposta S, Vergari M, Marceglia $S$, et al. Transcranial direct current stimulation improves recognition memory in Alzheimer disease. Neurology. 2008;71(7):493-8.

54. Boggio PS, Fregni F, Valasek C, Ellwood S, Chi R, Gallate J, et al. Temporal lobe cortical electrical stimulation during the encoding and retrieval phase reduces false memories. PLoS One. 2009;4(3):e4959.

55. Meinzer M, Lindenberg R, Phan MT, Ulm L, Volk C, Floel A. Transcranial direct current stimulation in mild cognitive impairment: behavioral effects and neural mechanisms. Alzheimers Dement. 2015;11(9):1032-40.

56. Andre S, Heinrich S, Kayser F, Menzler K, Kesselring J, Khader PH, et al. At-home tDCS of the left dorsolateral prefrontal cortex improves visual short-term memory in mild vascular dementia. J Neurol Sci. 2016;369:185-90

57. Marceglia S, Mrakic-Sposta S, Rosa M, Ferrucci R, Mameli F, Vergari M, et al. Transcranial direct current stimulation modulates cortical neuronal activity in Alzheimer's disease. Front Neurosci. 2016;10:134.

58. Liu CS, Herrmann N, Gallagher D, Rajji TK, Kiss A, Vieira D, et al. A pilot study comparing effects of bifrontal versus bitemporal transcranial direct current stimulation in mild cognitive impairment and mild alzheimer disease. J ECT. 2020;36(3):211-5.

59. Emonson MRL, Fitzgerald PB, Rogasch NC, Hoy KE. Neurobiological effects of transcranial direct current stimulation in younger adults, older adults and mild cognitive impairment. Neuropsychologia. 2019;125:51-61.

60. Liu CS, Rau A, Gallagher D, Rajji TK, Lanctot KL, Herrmann N. Using transcranial direct current stimulation to treat symptoms in mild cognitive impairment and Alzheimer's disease. Neurodegener Dis Manage. 2017:7(5):317-29. 
61. Hurley R, Machado L. Using tDCS priming to improve brain function: can metaplasticity provide the key to boosting outcomes? Neurosci Biobehav Rev. 2017:83:155-9.

62. Andrews SC, Hoy KE, Enticott PG, Daskalakis ZJ, Fitzgerald PB. Improving working memory: the effect of combining cognitive activity and anodal transcranial direct current stimulation to the left dorsolateral prefrontal cortex. Brain Stimul. 2011;4(2):84-9.

63. Mang CS, Snow NJ, Campbell KL, Ross CJ, Boyd LA. A single bout of high-intensity aerobic exercise facilitates response to paired associative stimulation and promotes sequence-specific implicit motor learning. J Appl Physiol. 2014;117(11):1325-36.

64. Singh AM, Neva JL, Staines WR. Acute exercise enhances the response to paired associative stimulation-induced plasticity in the primary motor cortex. Exp Brain Res. 2014;232(11):3675-85.

65. Manenti R, Brambilla M, Benussi A, Rosini S, Cobelli C, Ferrari C, et al. Mild cognitive impairment in Parkinson's disease is improved by transcranial direct current stimulation combined with physical therapy. Mov Disord. 2016:31(5):715-24.

66. Saleem M, Herrmann N, Dinoff A, Mielke MM, Oh PI, Shammi P, et al. A Lipidomics approach to assess the association between plasma Sphingolipids and verbal memory performance in coronary artery disease patients undertaking cardiac rehabilitation: a C18:0 signature for cognitive response to exercise. J Alzheimers Dis. 2017;60(3):829-41.

67. Sopova K, Gatsiou K, Stellos K, Laske C. Dysregulation of neurotrophic and haematopoietic growth factors in Alzheimer's disease: from pathophysiology to novel treatment strategies. Curr Alzheimer Res. 2014;11(1):27-39

68. Fritsch B, Reis J, Martinowich K, Schambra HM, Ji Y, Cohen LG, et al. Direct current stimulation promotes BDNF-dependent synaptic plasticity: potential implications for motor learning. Neuron. 2010;66(2):198-204

69. Wu YJ, Lin CC, Yeh CM, Chien ME, Tsao MC, Tseng P, et al. Repeated transcranial direct current stimulation improves cognitive dysfunction and synaptic plasticity deficit in the prefrontal cortex of streptozotocin-induced diabetic rats. Brain Stimul. 2017;10(6):1079-87.

70. Kim MS, Koo H, Han SW, Paulus W, Nitsche MA, Kim YH, et al. Repeated anodal transcranial direct current stimulation induces neural plasticity-associated gene expression in the rat cortex and hippocampus. Restor Neurol Neurosci. 2017;35(2):137-46.

71. Podda MV, Cocco S, Mastrodonato A, Fusco S, Leone L, Barbati SA, et al. Anodal transcranial direct current stimulation boosts synaptic plasticity and memory in mice via epigenetic regulation of Bdnf expression. Sci Rep. 2016;6:22180.

72. Zhang P, Liu ZT, He GX, Liu JP, Feng J. Low-voltage direct-current stimulation is safe and promotes angiogenesis in rabbits with myocardial infarction. Cell Biochem Biophys. 2011;59(1):19-27.

73. Religa P, Cao R, Religa D, Xue Y, Bogdanovic N, Westaway D, et al. VEGF significantly restores impaired memory behavior in Alzheimer's mice by improvement of vascular survival. Sci Rep. 2013;3:2053.

74. Guo T, Fang J, Tong ZY, He S, Luo Y. Transcranial direct current stimulation ameliorates cognitive impairment via modulating oxidative stress, inflammation, and autophagy in a rat model of vascular dementia. Front Neurosci. 2020;14:28.

75. Kim YW, Byzova TV. Oxidative stress in angiogenesis and vascular disease. Blood. 2014;123(5):625-31.

76. Siuda J, Patalong-Ogiewa M, Zmuda W, Targosz-Gajniak M, Niewiadomska E, Matuszek I, et al. Cognitive impairment and BDNF serum levels. Neurol Neurochir Pol. 2017;51(1):24-32.

77. Deininger MH, Fimmen BA, Thal DR, Schluesener HJ, Meyermann R. Aberrant neuronal and paracellular deposition of endostatin in brains of patients with Alzheimer's disease. J Neurosci. 2002;22(24):10621-6.

78. Diagnostic and statistical manual of mental disorders 5 th edn Arlington: American Psychiatric Association; 2013.

79. Molloy DW, Standish TI. A guide to the standardized mini-mental state examination. Int Psychogeriatr. 1997;9(Suppl 1):87-94 discussion 143-50.

80. CSEP. The physical activity readiness questionnaire for everyone. 2011.

81. Nelson ME, Rejeski WJ, Blair SN, Duncan PW, Judge JO, King AC, et al. Physical activity and public health in older adults: recommendation from the American College of Sports Medicine and the American Heart Association. Circulation. 2007;116(9):1094-105.

82. Tremblay MS, Warburton DE, Janssen I, Paterson DH, Latimer AE, Rhodes RE, et al. New Canadian physical activity guidelines. Appl Physiol Nutr Metab. 2011;36(1):36-46 7-58.

83. Langhammer B, Bergland A, Rydwik E. The importance of physical activity exercise among older people. Biomed Res Int. 2018;2018:7856823.

84. Garrido GE, Furuie SS, Buchpiguel CA, Bottino CM, Almeida OP, Cid CG, et al. Relation between medial temporal atrophy and functional brain activity during memory processing in Alzheimer's disease: a combined MRI and SPECT study. J Neurol Neurosurg Psychiatry. 2002;73(5):508-16.

85. Brunoni AR, Nitsche MA, Bolognini N, Bikson M, Wagner T, Merabet $L$, et al. Clinical research with transcranial direct current stimulation (tDCS): challenges and future directions. Brain Stimul. 2012;5(3):175-95.

86. Paulus W. Transcranial direct current stimulation (tDCS). Suppl Clin Neurophysiol. 2003;56:249-54.

87. Godin G, Shephard RJ. A simple method to assess exercise behavior in the community. Can J Appl Sport Sci. 1985;10(3):141-6.

88. Freitas S, Simoes MR, Alves L, Santana I. Montreal cognitive assessment: validation study for mild cognitive impairment and Alzheimer disease. Alzheimer Dis Assoc Disord. 2013;27(1):37-43.

89. Trzepacz PT, Hochstetler H, Wang S, Walker B, Saykin AJ. Relationship between the Montreal cognitive assessment and Mini-mental state examination for assessment of mild cognitive impairment in older adults. BMC Geriatr. 2015;15:107.

90. Costa AS, Reich A, Fimm B, Ketteler ST, Schulz JB, Reetz K. Evidence of the sensitivity of the MoCA alternate forms in monitoring cognitive change in early Alzheimer's disease. Dement Geriatr Cogn Disord. 2014;37(1-2):95-103.

91. Chau SA, Herrmann N, Eizenman M, Chung J, Lanctot KL. Exploring visual selective attention towards novel stimuli in Alzheimer's disease patients. Dement Geriatr Cogn Disord Extra. 2015;5(3):492-502.

92. Rosen WG, Mohs RC, Davis KL. A new rating scale for Alzheimer's disease. Am J Psychiatry. 1984;141(11):1356-64.

93. Tsai CL, Pan CY, Chen FC, Tseng YT. Open- and closed-skill exercise interventions produce different neurocognitive effects on executive functions in the elderly: a 6-month randomized, controlled trial. Front Aging Neurosci. 2017;9:294.

94. Liu-Ambrose T, Best JR, Davis JC, Eng JJ, Lee PE, Jacova C, et al. Aerobic exercise and vascular cognitive impairment: a randomized controlled trial. Neurology. 2016;87(20):2082-90.

95. Arevalo-Rodriguez I, Smailagic N, Roque IFM, Ciapponi A, SanchezPerez E, Giannakou A, et al. Mini-mental state examination (MMSE) for the detection of Alzheimer's disease and other dementias in people with mild cognitive impairment (MCl). Cochrane Database Syst Rev. 2015:(3):CD010783.

96. Cummings JL, Mega M, Gray K, Rosenberg-Thompson S, Carusi DA, Gornbein J. The neuropsychiatric inventory: comprehensive assessment of psychopathology in dementia. Neurology. 1994;44(12):2308-14.

97. Lai CK. The merits and problems of neuropsychiatric inventory as an assessment tool in people with dementia and other neurological disorders. Clin Interv Aging. 2014;9:1051-61.

98. Ganguli M. Depression, cognitive impairment and dementia: why should clinicians care about the web of causation? Indian J Psychiatry. 2009;51(Suppl 1):S29-34.

99. de Oliveira FF, Wajman JR, Bertolucci PH, Chen ES, Smith MC. Correlations among cognitive and behavioural assessments in patients with dementia due to Alzheimer's disease. Clin Neurol Neurosurg. 2015;135:27-33.

100. Brodaty $\mathrm{H}$, Connors $\mathrm{MH}, \mathrm{Xu} J$, Woodward M, Ames D. The course of neuropsychiatric symptoms in dementia: a 3-year longitudinal study. J Am Med Dir Assoc. 2015;16(5):380-7.

101. Parikh SM, Mammoto T, Schultz A, Yuan HT, Christiani D, Karumanchi SA, et al. Excess circulating angiopoietin-2 may contribute to pulmonary vascular leak in sepsis in humans. PLoS Med. 2006;3(3):e46.

102. Golledge J, Clancy P, Hankey GJ, Yeap BB, Norman PE. Serum endostatin concentrations are higher in men with symptoms of intermittent claudication. Dis Markers. 2014;2014:298239.

103. Mateo I, Llorca J, Infante J, Rodriguez-Rodriguez E, Fernandez-Viadero C, Pena N, et al. Low serum VEGF levels are associated with Alzheimer's disease. Acta Neurol Scand. 2007:116(1):56-8. 
104. Dinoff A, Herrmann N, Swardfager W, Liu CS, Sherman C, Chan S, et al. The effect of exercise training on resting concentrations of peripheral brain-derived Neurotrophic factor (BDNF): a Meta-analysis. PLoS One. 2016;11(9):e0163037.

105. Dinoff A, Herrmann N, Swardfager W, Lanctot KL. The effect of acute exercise on blood concentrations of brain-derived neurotrophic factor in healthy adults: a meta-analysis. Eur J Neurosci. 2017:46(1):1635-46.

106. Morland C, Andersson KA, Haugen OP, Hadzic A, Kleppa L, Gille A, et al. Exercise induces cerebral VEGF and angiogenesis via the lactate receptor HCAR1. Nat Commun. 2017;8:15557.

107. Sponder M, Fritzer-Szekeres M, Marculescu R, Litschauer B, StrametzJuranek J. Physical inactivity increases endostatin and osteopontin in patients with coronary artery disease. Heart Vessel. 2016;31(10):1603-8.

108. Hoier B, Walker M, Passos M, Walker PJ, Green A, Bangsbo J, et al. Angiogenic response to passive movement and active exercise in individuals with peripheral arterial disease. J Appl Physiol. 2013;115(12):1777-87.

109. Cass SP. Alzheimer's disease and exercise: a literature review. Current sports medicine reports. 2017;16(1):19-22.

110. Cruz Gonzalez P, Fong KNK, Chung RCK, Ting KH, Law LLF, Brown T. Can Transcranial direct-current stimulation alone or combined with cognitive training be used as a clinical intervention to improve cognitive functioning in persons with mild cognitive impairment and dementia? A systematic review and meta-analysis. Front Hum Neurosci. 2018;12:416.

111. Park J, Oh Y, Chung K, Kim KJ, Kim CO, Park JY. Effect of home-based transcranial direct current stimulation (tDCS) on cognitive function in patients with mild cognitive impairment: a study protocol for a randomized, double-blind, cross-over study. Trials. 2019;20(1):278.

112. Lefebvre S, Liew SL. Anatomical parameters of tDCS to modulate the motor system after stroke: a review. Front Neurol. 2017;8:29.

\section{Publisher's Note}

Springer Nature remains neutral with regard to jurisdictional claims in published maps and institutional affiliations.

Ready to submit your research? Choose BMC and benefit from:

- fast, convenient online submission

- thorough peer review by experienced researchers in your field

- rapid publication on acceptance

- support for research data, including large and complex data types

- gold Open Access which fosters wider collaboration and increased citations

- maximum visibility for your research: over $100 \mathrm{M}$ website views per year

At BMC, research is always in progress.

Learn more biomedcentral.com/submissions 